\title{
Does superhigh-energy cosmic-ray coplanarity contradict LHC data?
}

\author{
R. A. Mukhamedshin ${ }^{\mathrm{a}}(\mathbb{C}$ \\ Institute for Nuclear Research, Russian Academy of Sciences, Moscow 117312, Russia
}

Received: 28 May 2018 / Accepted: 9 May 2019 / Published online: 24 May 2019

(C) The Author(s) 2019

\begin{abstract}
One of phenomena observed in cosmic-ray experiments at $E_{0} \gtrsim 10^{16} \mathrm{eV}(\sqrt{s} \gtrsim 5 \mathrm{TeV})$ is a so-called coplanarity of most energetic subcores of $\gamma$-ray-hadron families. This effect requires appearance of some coplanar generation of most energetic particles in hadron-nucleus interactions. To improve research for this phenomenon, a few versions of the new phenomenological FANSY 2.0 model are designed. This model makes it possible to simulate interactions via both traditional and coplanar hadron generation modes. The model reproduces ALICE, ATLAS, CMS, TOTEM data on lowrapidity particle generation, LHCf's high- $x_{\mathrm{F}}$ and high- $\eta$ data on $\gamma$-rays and neutron-like hadrons as well as low-energy data on generation of stable and resonance hadrons, lightquark, strange and charmed particles. Features of coplanar particle generation of hadrons at superhigh energies are discussed. A test experiment at the LHC is proposed.
\end{abstract}

\section{Introduction}

A number of models of high-energy hadron-nucleus interactions is concurrently applied in cosmic-ray experiments. However, none of the models can reproduce the complete set of experimental extensive air shower (EAS) data with some sufficient accuracy (see [1], e.g.).

Besides, some results obtained in mountain-based and stratospheric X-ray-emulsion chamber (XREC) experiments are not explained yet. The present paper considers one of these phenomena, namely, a strong azimuthal effect that manifests itself in the form of a tendency to a coplanarity of most energetic subcores of so-called $\gamma$-ray-hadron families, i.e., groups of high-energy ( $E \gtrsim n \cdot 1 \mathrm{TeV}$ ) particles ( $\gamma$-rays, $\mathrm{e}^{ \pm}$, hadrons) in cores of relatively young extensive air showers, i.e., showers which do not reach the maximum of their

\footnotetext{
a e-mail: rauf_m@mail.ru
}

development, and the number of interactions of most energetic particles in these showers is much less than the average one. These showers are initiated mainly by protons and light nuclei of the primary cosmic radiation (PCR) with energies $E_{0} \gtrsim 10^{16} \mathrm{eV}(\sqrt{s} \gtrsim 5 \mathrm{TeV})$.

The effect was found by the Pamir Collaboration [2-6] and observed later in other experiments [7-11].

There is a point of view that this effect is not more than a giant fluctuation. This opinion is mainly based, on the one hand, on the fact that collider experiments do not inform us on such interactions. On the other hand, it is caused by desultory and fragmentary awareness based, as a rule, on separated results presented by different experimental groups with different XREC types, namely, so-called "carbon", "lead", "iron" (C-, Pb-, Fe-XREC) with a lateral resolution of the order of $\gtrsim 100 \mu \mathrm{m}$.

However, to analyze this problem, it is necessary to take into account the following circumstances.

First, LHC experiments cannot give non-inclusive data on high- $x_{\mathrm{F}}$ multiple hadron generation.

Secondly, five independent sets of experimental data were accumulated, namely, data of Pamir Collaboration's C- and $\mathrm{Pb}$-XRECs on $\gamma$-ray families with energies $\sum E_{\gamma} \geq 700$ $\mathrm{TeV}$ and data of Mt.Canbala Collaboration's Fe-XRECs $\left(\sum E_{\gamma} \geq 500 \mathrm{TeV}\right)$.

Besides, only two stratospheric events (the Strana and $J F 2 a f 2$ ) with $\sum E_{\gamma}>1 \mathrm{PeV}$ have been detected at very high altitudes, and both of these events demonstrate very high coplanarity of most energetic particles (MEP). It should be noted that the features of the stratospheric events are much more sensitive to the first interactions of PCR particles (due to a much smaller thickness of the atmospheric layer above the XREC detectors in each of these experiments), whereas events at mountain levels are strongly contaminated by secondary interactions of cascade hadrons.

All these data are related to hadron-nucleus interaction energies $E_{0} \gtrsim 10^{16} \mathrm{eV}$. The parameter 
Table 1 Summary of experimental data. Columns: 1, Data set and type of used XRECs; 2-3, Criteria for selection of coplanar events; 4, Number of coplanar families, $N_{\exp }^{\text {copl }}$, and total family number $N_{\text {tot }} ; 5$, Frac- tion of coplanar families, $F_{\exp }^{\mathrm{copl}} .6$, References. 7, Simulated background fraction of coplanar $\gamma$-ray families, $F_{\text {sim }}^{\text {copl }}[14]$

\begin{tabular}{|c|c|c|c|c|c|c|}
\hline \multirow[t]{2}{*}{ Experimental data set } & \multicolumn{2}{|c|}{ Selection criteria } & \multirow[t]{2}{*}{$N_{\text {exp }}^{\operatorname{copl}} / N_{\text {tot }}$} & \multirow[t]{2}{*}{$F_{\text {exp }}^{\text {copl }}$} & \multirow[t]{2}{*}{ Ref. } & \multirow[t]{2}{*}{$F_{\text {sim }}^{\mathrm{copl}}$} \\
\hline & $\overline{\lambda_{N}}$ & $\sum E_{\gamma}$ & & & & \\
\hline 1 & 2 & 3 & 4 & 5 & 6 & 7 \\
\hline $\operatorname{Pamir}(\mathrm{Pb})$ & $\lambda_{4} \geq 0.8$ & $\sum E_{\gamma} \geq 700 \mathrm{TeV}$ & $6 / 14$ & $0.43 \pm 0.13$ & {$[4]$} & $0.041 \pm 0.004$ \\
\hline Pamir (C) & $\lambda_{4} \geq 0.8$ & $\sum E_{\gamma} \geq 700 \mathrm{TeV}$ & $5 / 35$ & $0.15 \pm 0.05$ & {$[6]$} & $0.049 \pm 0.004$ \\
\hline Mt.Kanbala $(\mathrm{Fe})$ & $\lambda_{3} \geq 0.8$ & $\sum E_{\gamma} \geq 500 \mathrm{TeV}$ & $6 / 12$ & $0.5 \pm 0.13$ & [7] & $0.180 \pm 0.010$ \\
\hline the Strana & $\lambda_{4}=0.99$ & $\sum E_{\gamma} \simeq 1500 \mathrm{TeV}$ & 1 & 1 & {$[15,16]$} & $0.0026 \pm 0.0003$ \\
\hline the $J F 2 a f 2$ & $\lambda_{4}=0.998$ & $\sum E_{\gamma} \simeq 1500 \mathrm{TeV}$ & 1 & 1 & {$[10]$} & $(9 \pm 3) \times 10^{-4}$ \\
\hline
\end{tabular}

$\lambda_{N}=\sum_{i \neq j \neq k}^{N} \cos 2 \varphi_{i j}^{k} /(N(N-1)(N-2))$,

was proposed by the Pamir Collaboration [2] to calculate the degree of a so-called alignment of $N$ point-like tracks on a target plane that have appeared due to falling of MEPs to the XREC. Here $\varphi_{i j}^{k}$ is the angle between two vectors, connecting the $k$-th point with the $i$-th and $j$-th points. The summation is performed over all possible combinations. The parameter $\lambda_{N}$ is equal to unity for $N$ points aligned along a straight line and $\lambda_{N} \approx-1 /(N-1)$ in the isotropic cases.

Table 1 shows types of experimental data sets and used XRECs; criteria used to select experimental coplanar (or aligned) events, i.e., values of $\lambda_{N}$ and energy $\sum E_{\gamma}$ of $\gamma$ ray families; number of aligned families, $N_{\exp }^{\mathrm{copl}}$, and total family number $N_{\text {tot }}$; experimental fraction of aligned families, $F_{\text {exp }}^{\text {copl }}$, observed in each experimental data set; corresponding references (Columns $1-6$, respectively). Background fractions of aligned $\gamma$-ray families, $F_{\text {sim }}^{\text {copl }}$, simulated using the QGSM-based FANSY 1.0 model [14] are given in Column 7.

It is obvious that although the statistical security of the data of each of the ground-based experiments is not too high, each of the data sets demonstrates some excess over the corresponding simulated value. Both stratospheric events are particularly unique in terms of both energy and extreme alignment of MEP tracks.

This phenomenon under consideration cannot be studied in EAS experiments due to a poor coordinate and energy resolution of detectors aimed at measurements of the total lowenergy charged-particle number, but not the narrow highenergy structure of the central EAS core.

The phenomenon is related to energies which were unreachable with the use of previous generation of accelerators. However, the LHC makes it possible to study this problem.
The probability for the total set of these experimental results to be produced by EAS fluctuations is rather negligible (less than $\sim 10^{-10}$ ) [12-14].

The phenomenon was initially interpreted as a manifestation of coplanar generation of most energetic particles in the fragmentation range $\left(x_{L a b}=E / E_{0} \gtrsim 0.01-0.05\right)$ characterized by relatively large transverse momenta, $p_{t}^{\text {copl }}>1$ $\mathrm{GeV} / \mathrm{c}$. Besides, it was shown with using a nuclear-emulsion detector that $p_{t}^{\text {copl }}$ can rise up to $\sim n \times 10 \mathrm{GeV} / \mathrm{c}$ as early as at $E_{0} \sim 10^{16} \mathrm{eV}[15,16]$. However, it should be emphasized that this result should be interpreted with caution because of some methodological uncertainties of this experiment.

As regards $\gamma$-ray families observed in ground-based experiments, a more accurate measurement or, at least, an estimation of the MEPs' average $p_{t}^{\text {copl }}$ in aligned events was not carried out in reality at the time of realization of the above-mentioned experiments due to difficulties in simulation of XREC response.

However, one can say that the average ratio of the MEPs' transverse momentum components lying in the coplanarity plane, $\left\langle\mathbf{p}_{t}^{\text {copl }}\right\rangle$, to the $\mathbf{p}_{t}$ components directed normally to the coplanarity plane, $\left\langle\mathbf{p}_{t}^{\text {norm }}\right\rangle$, is large, i.e. $\left\langle p_{t}^{\text {copl }} / p_{t}^{\text {norm }}\right\rangle \sim 10$ [4].

In general, the coplanarity phenomenon is [12]

- associated with most energetic secondary particles;

- not described by traditional QGS models and QCD approach;

- characterized by a relatively large cross section that is comparable with traditional inelastic proton-air highenergy interaction cross section estimations, $\sigma_{\text {prod }}^{p \text {-air }}$.

Just a few theoretical ideas were proposed to explain this phenomenon, namely,

- conservation of the angular momentum of a relativistic fast-rotating quark-gluon string (QGS) stretched between colliding hadrons [17]; 
- semihard double inelastic diffraction (SHDID) [18], which assumes the coplanarity to be a result of some QGS tension in the diffraction cluster between a semihardly scattered constituent quark and other spectator quarks of the projectile hadron;

- projectile's diquark breaking [19];

- appearance of very-high-spin leading systems [20,21];

- the most extraordinary and speculative assumption of a connection between this phenomenon and the hypothesis of "crystal world" with latticed and anisotropic spatial dimensions [22].

At least, it cannot be excluded that this phenomenon is a signature of some coherent Cherenkov-like hadron radiation at ultrahigh-energies [23].

It should be noted that the first four hypotheses include large transverse momenta as an almost mandatory element while the "crystal world" idea implies a gradual reduction of space dimensionality from three to two dimensions at sufficiently high energies. It entails the $\mathbf{p}_{t}$ localization in some plane and a reduction of $\mathbf{p}_{t}$ components directed perpendicularly to this plane.

Unfortunately, all of these ideas are not quantitatively developed, especially, the last one.

It looks like that in this situation we can use both largeand normal- $p_{t}$ approaches to study this phenomenon.

While considering the angular-momentum conservation approach with respect to generation of secondary particles, Fermi [24] many years ago has neglected it “...to simplify the mathematics". Hagedorn [25] noted later that "this whole question, though of practical importance, seems to be still not understood. ... it seems most reasonable to disregard angular momentum at all". Unfortunately, this tendency dominates until now, although energies under consideration increased since Fermi and Hagedorn's time by many orders of magnitude. As a result, to analyze the observed coplanarity phenomenon, we can construct only quite arbitrary models without any theoretical basis.

In any case, the experimental result shows that some characteristics of strong "forward-physics" interactions at superhigh energies (at least, MEP generation coplanarity) may not be reproducible with a concept presented by such traditional quark-gluon string-based models (QGSM) as QGSJETII-03 [26,27], DPMJET 3.04 [28], SIBYLL 2.1 [29-31], EPOS 1.99 [32,33], PYTHIA 8.145 [34], used in the cosmic-ray physics.

The coplanar generation of most energetic particles could be phenomenologically reproduced in the framework of two concepts, namely, (a) initial generation of quark-gluon strings with the following multiparticle production; (b) initial generation of an energetic leading system (like diffraction clusters, e.g.) with a lower multiplicity and higher average energies of particles. In this work, the first approach is only considered.

It should be noted that the second way must be considered separately as the contribution of diffraction processes into phenomena observed in cosmic-ray experiments is more significant than their formal contribution into the inelastic cross section.

The energy range corresponding to the coplanar generation of MEPs is studied at the LHC that makes it possible to test this phenomenon and, maybe, to make this problem more clear.

It would be very interesting to search for a possible relation of this phenomenon with the "ridge" effect found by the CMS Collaboration [35]. The "ridge" effect shows a two-particle long-range azimuthal-angle correlation at $\phi \approx 0$ and $\Delta \eta \gtrsim$ 4. At first glance, these two phenomena could be somehow related. However, collider and cosmic-ray experiments are carried out with completely different event-selection criteria (very high particle multiplicity in the central pseudorapidity range in the case of the "ridge" effect and a few high- $x_{\mathrm{F}}$ MEPs in coplanar $\gamma$-ray families). As a result, at present one can neither exclude nor confirm a relationship between these effects.

To study this problem, the phenomenological model FANSY $1.0[13,14]$ has been developed a dozen years ago (before the launch of the LHC). It described main characteristics of $\gamma$-ray families (for instance, their intensity and mean transversal size) and helped to understand general features of the coplanar experimental events. However, FANSY 1.0 cannot properly describe some observed features of $\gamma$-ray families, namely, their average transversal size [36]. Besides, FANSY 1.0 cannot properly describe such LHC data as, for example, LHCf results on high- $x_{\mathrm{F}} \gamma$-rays ([38], e.g.) and neutron-like hadrons $\left(n, \bar{n}\right.$, neutral kaons, $\Lambda^{0}, \bar{\Lambda}^{0}$ etc.) which are really important for cosmic ray experiments.

To improve this situation, a new phenomenological model FANSY 2.0 [37] is designed. The model makes it possible to simulate QGSM-based traditional and coplanar particle generation (QGSJ and QGSCPG versions, respectively) with a higher accuracy of description of experimental data as compared with FANSY 1.0. Both the FANSY 2.0 versions reproduce high- $x_{\mathrm{F}}$ and high- $\eta$ data on $\gamma$-rays and neutron-like hadrons accumulated by the LHCf experiment, as well as ALICE, ATLAS, CMS, TOTEM data on generation of stable particles and resonances, mesons and baryons, light-quarks, strange and charmed particles. In addition, FANSY 2.0 reproduces low-energy data on generation of stable particles and resonances, strange and charmed particles and so on.

One can summarize the following key findings.

- a coplanarity of most energetic subcores of $\gamma$-ray-hadron families is observed in XREC experiments; 
- the phenomena is not described by traditional QGS models and QCD approach as well as fluctuations of EAS development;

- the effect is characterized by a relatively large cross section that is comparable with $\sigma_{\text {prod }}^{\text {p-air }}$;

- a few qualitative theoretical ideas were proposed to explain this phenomenon;

- a phenomenological model could be only used to study main features of this phenomenon.

This paper is organized as follows. Section 2 presents a short discussion on simulation problems related to the primary concept of coplanarity origin and describes shortly the FANSY 2.0 QGSCPG version with coplanar particle generation (CPG). Section 3 compares some experimental and simulated results on interactions features. Problems related to the proposed LHC experiment are considered in Sect. 4. Final comments are given in Conclusion.

More details of comparison of experimental data with results of simulation with the basic FANSY 2.0 QGSJ version are given in Ref. [37].

\section{Coplanarity simulation}

\subsection{Coplanarity concepts}

To analyze the coplanarity problem, first of all, the hypothesis of conservation of the angular momentum of relativistic fast-rotating quark-gluon string (QGS) stretched between colliding hadrons [17] is applied to develop the FANSY 2.0 QGSCPG version which simulates coplanarization of MEPs generated in nondiffraction processes.

Simulations using FANSY 2.0 QGSCPG tentative versions shows the following fundamental problem.

The original concept exploited by FANSY $1.0[13,14]$ qualitatively explains the observed coplanarity of longitudinal momenta of MEPs with assuming their high transverse momenta, $p_{t}^{\text {copl }}$, in a coplanarity plane. However, in this case a significant $p_{t}$ growth suppresses $d \sigma / d y$ and $d \sigma / d \eta$ cross sections of hadron generation at highest $|y|$ and $|\eta|$ values and creates robust peaks at $2 \lesssim|\eta| \lesssim 4$ which are contrary to LHC data (Fig. 1). This was an unsolvable problem for all the considered QGSCPG versions based on the initial high- $p_{t}^{c o p l}$ coplanarity concept.

However, the coplanarity is actually observed in cosmicray experiments. Is it possible to reconcile this result with the LHC data?

Simulation shows that some agreement of LHC data and idea of coplanar generation becomes real only using a new concept of coplanarity origin, namely, assuming some decrease of particles' transverse momentum components directed normally to the coplanarity plane. This decrease

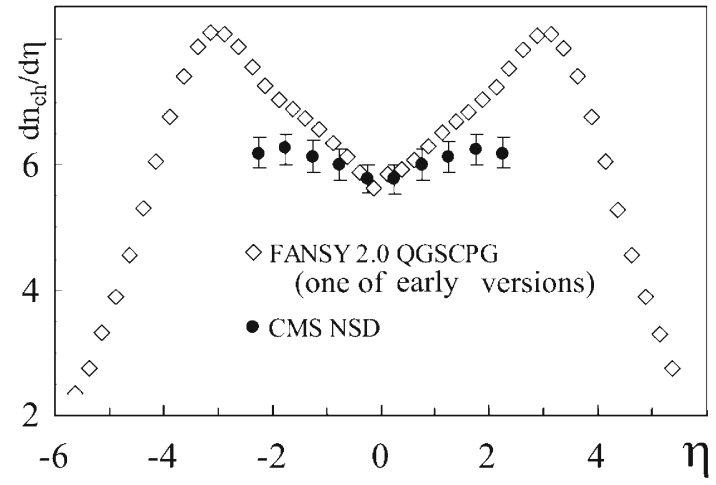

Fig. 1 Peaks in $d \sigma / d \eta$ distribution originated by growth of transverse momenta of most energetic particles in high- $p_{t}^{\text {copl }}$-concept CPG versions

takes place so that the average absolute $p_{t}$ values in any $\eta$ bin do not change significantly. Some weak increase of $p_{t}$ values cannot be excluded.

While introducing azimuth effects, the algorithm keeps transverse momenta of each of MEPs constant. In this paper, some turning of particle's $p_{t}$ with no change of its absolute value is exploited. As a result, corresponding $p_{t}$ distributions are unchanged.

Figure 2 shows three versions of observed tracks of particles, generated in the same imaginary interaction and arriving on a target plane placed at some distance from the interaction point. Most energetic particles are shown with large black symbols. One can see cases of traditional QGSM-like interaction (left), primary-concept coplanar interaction with increased $p_{t}^{\text {copl }}$ (middle), and new-concept coplanar interaction with traditional average $p_{t}^{c o p l}$ magnitudes (right). The geometric scale is given in arbitrary units.

\subsection{Coplanarity simulation}

As it is mentioned in Sect. 2.1, the hypothesis of conservation of the angular momentum of a relativistic fast-rotating quarkgluon string (QGS) stretched between colliding hadrons [17] is applied to develop the QGSCPG version which simulates coplanarization of the main bulk of secondary particles.

All interaction characteristics (excluding azimuthal ones) simulated with FANSY 2.0 QGSJ and QGSCPG versions, are similar. With decreasing energy, both the QGSJ and QGSCPG versions gradually become identical at $\sqrt{s} \lesssim 2$ $\mathrm{TeV}$.

Simulation of CPG processes is realized as follows. All characteristics of particles in the QGSCPG version are primarily simulated with the traditional way, i.e., applying the QGSJ option. After this, hadron longitudinal momenta are listed in decreasing order, i.e., $p_{\max }>p_{1}>p_{2}>p_{3}>$ $p_{4} \ldots p_{n}>-p_{\max }$. The corresponding rapidity values of 


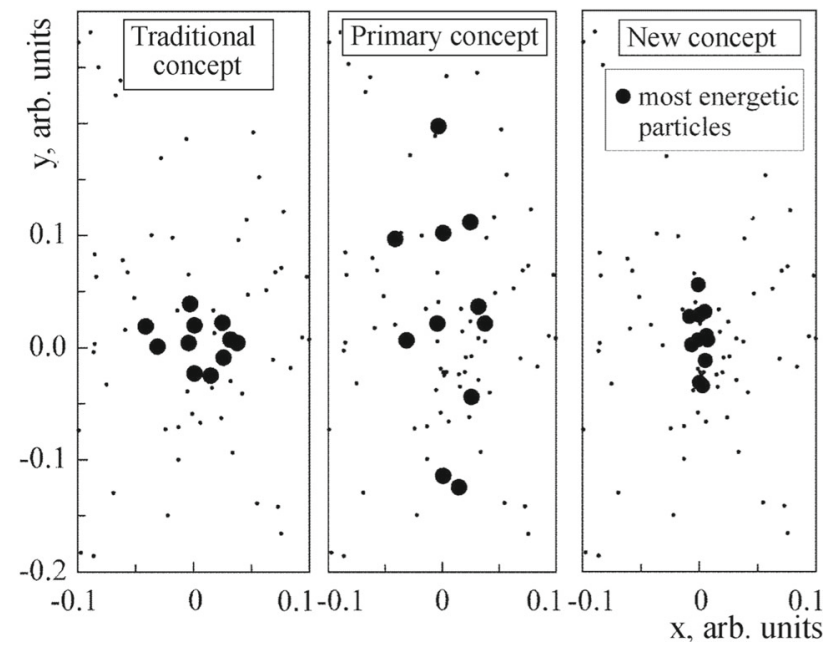

Fig. 2 Tracks of particles, generated in the same interaction, on a target plane in cases of traditional particle generation (left), coplanar particle generation within the primary concept of increased $p_{t}$ (middle) and coplanar particle generation within the new concept of traditional $p_{t}$ (right). Most energetic particles are shown with large filled circles

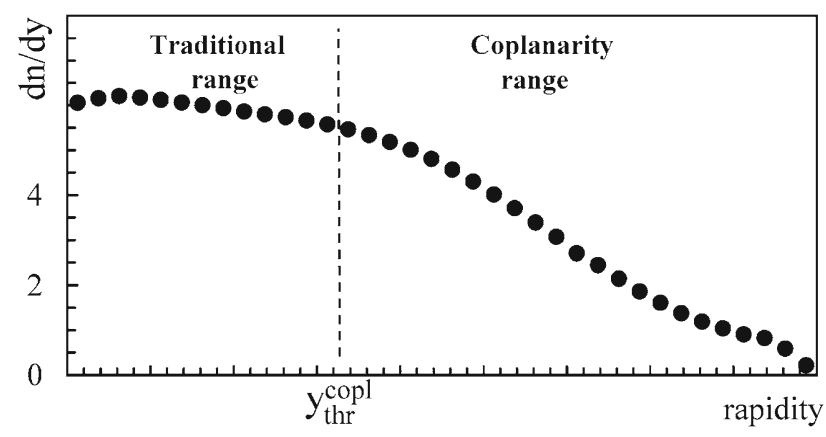

Fig. 3 Qualitative scheme of traditional and coplanarity ranges of $d n_{c h} / d y$ distribution

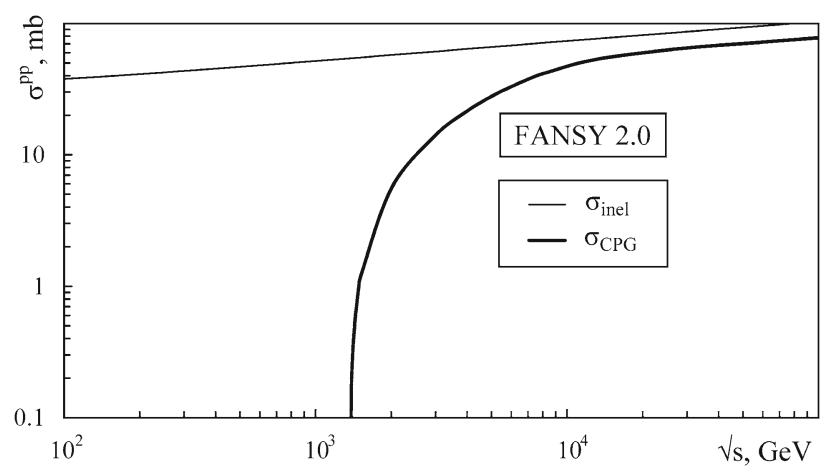

Fig. 4 Energy dependence of cross section of coplanarization process simulation, $\sigma_{\text {inel }}^{\mathrm{CPG}}(s)$ these hadrons are denoted by $y_{1}, y_{2}, y_{3}, y_{4} \ldots y_{n}$. In $p p$ interactions, $p_{\max }=\sqrt{s / 4-m_{p}^{2}}$.

After this, the so-called "coplanarization" procedure is implemented for MEPs in the coplanarity range (i.e., $|y|>$ $y_{t h r}^{c o p l}$ ) of the hadron rapidity distribution. Figure 3 shows a schematic drawing of the traditional and coplanarity ranges of $d n / d y$ distributions.

While beginning from the most energetic particle, transversal momenta of high-rapidity particles with $|y|>y_{\text {thr }}^{c o p l}$ are coplanarized with a probability $w^{\text {copl }}(s)=\sigma_{\text {inel }}^{\mathrm{CPG}} / \sigma_{\text {inel }}$, if the summary energy of secondary particles, $\sqrt{s_{\text {eff }}}$, is higher than a fixed value, $\sqrt{s_{t h r}^{\mathrm{CPG}}}$ (although real observable effects appear at significantly higher $\sqrt{s_{\text {eff }}}$ values).

The position of the coplanarity plane is determined by the longitudinal momenta of the interacting projectile hadrons and the summary transverse momentum of two most energetic hadrons with momenta $p_{1}$ and $p_{n}$ after the interaction.

An algorithm rotates the transverse-momentum vector of all the hadrons with $|y|>y_{t h r}^{c o p l}$ towards the coplanarity plane along the shortest path and stops this procedure at $|y|<y_{t h r}^{c o p l}$. The azimuth-angle distribution of transversal momenta of most energetic particles with rapidity $y$ relative to the coplanarity plane obeys a Gaussian distribution with a standard deviation of $\sigma_{\Delta \phi}^{c o p l}(y)=\sigma_{0}^{c o p l} \cdot\left(y_{2} /|y|\right)$. As a result, $\sigma_{\Delta \phi}^{c o p l}(y)$ is minimum for large-rapidity hadrons and increases with decreasing rapidity, i.e., the coplanarity is suppressed with decreasing hadron rapidities.

Here $y_{t h r}^{c o p l}=y_{2}-\Delta y^{c o p l}$, where $\Delta y^{c o p l}$ is a free parameter.

The value of $y_{\text {thr }}^{\text {copl }}$ varies from $\sim 2$ to $\sim 5$ for hadrons with $p_{z}>0$ even at a constant $\Delta y^{\text {copl }}$ due to fluctuations of $\sqrt{s_{\text {eff }}}$ and hadron rapidity values. Similar procedure is carried out for hadrons with $p_{z}<0$. If the "ridge" effect [35] is associated with the coplanar generation of MEPs, then $y_{t h r}^{c o p l}$ seems to be of $\sim 2-3$.

Figure 4 shows the energy dependence of cross section of the coplanarization process simulation, $\sigma_{i n e l}^{\mathrm{CPG}}(s)$, as well as inelastic cross section, $\sigma_{\text {inel }}(s)$, in $p p$ interactions. Observable results are determined by values of $\sqrt{s_{t h r}^{\mathrm{CPG}}}, \Delta y^{\text {copl }}$, $\sigma_{0}^{c o p l}$, and interaction energy. One can see that $\sigma_{0}^{c o p l}$ is characterized with an energy threshold of $\sqrt{s} \approx 1.4 \mathrm{TeV}$ and fast growth up to $0.89 \sigma_{\text {inel }}$ at $\sqrt{s} \gtrsim 10 \mathrm{TeV}$.

All the results given in this paper are obtained at $\sqrt{s_{t h r}^{\mathrm{CPG}}}=$ $250 \mathrm{GeV} / \mathrm{c}, \Delta y^{\mathrm{copl}}=4, \sigma_{0}^{\mathrm{copl}}=0.025 \mathrm{rad}$ and $\sqrt{\mathrm{s}}=7$ $\mathrm{TeV}$. The average $y_{2}$ value, $\left\langle y_{2}\right\rangle$, is actually equal to 7.15. As a result, $\left\langle y_{t h r}^{c o p l}\right\rangle=3.15$.

Obviously, the described algorithm of the coplanarization procedure is highly arbitrary, has not any theoretical basis, and could be used as a phenomenological zero-order approx- 


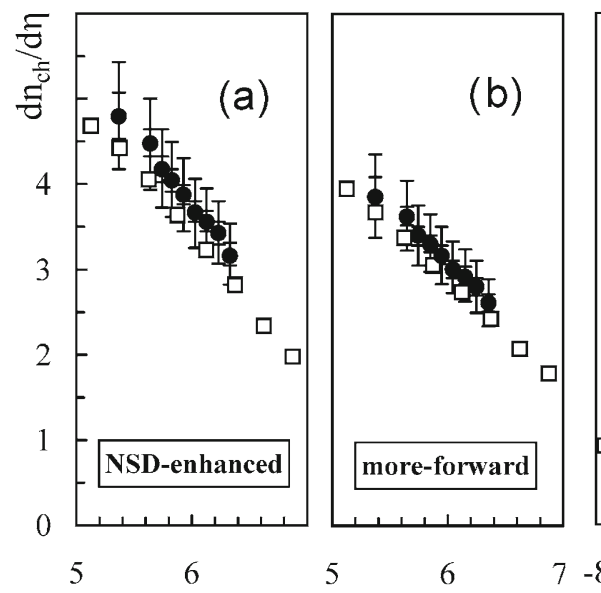

Fig. 5 CMS+TOTEM [41] and FANSY 2.0 QGSCPG's $d n_{c h} / d \eta$ data for events selected at $\sqrt{s}=8 \mathrm{TeV}$ under the following requirements. "NSD-enhanced" data: $n_{c h} \geq 1$ in the ranges $-6.5<\eta<-5.3$ and $5.3<\eta<6.5$ (a); "more-forward" data: $n_{c h} \geq 1$ in the ranges $-6.5<\eta<-5.3$ or $5.3<\eta<6.5$ (b); "SD-enhanced" data (lower

imation tool only to estimate the influence of $\mathrm{CPG}$ processes on observed interaction characteristics.

\section{LHC data and FANSY 2.0 QGSCPG results}

Experimental LHC data are mainly related to the central low-rapidity range. However, the coplanarity phenomenon is associated with most energetic particles in the cosmic-rayinitiated EAS core at the initial development stage, i.e., close to first interaction of the primary particle.

Therefore, only experimental high- $\eta$ and high- $x_{\mathrm{F}}$ LHCf data [38-40] at $\sqrt{s}=7 \mathrm{TeV}$ on neutrons, $\bar{n}, \gamma$-rays mainly with a relatively low contribution of unstable $K_{L}^{0}$ mesons and $\Lambda^{0}, \bar{\Lambda}^{0}$ hyperons as well as high- $\eta$ CMS+TOTEM $d n_{c h} / d \eta$ data at $\sqrt{s}=8 \mathrm{TeV}$ [41] are compared with FANSY 2.0 results in Sect. 3 .

More detailed comparison of LHC data and results of simulation of $p p$ interactions with the FANSY 2.0 QGSJ in a wide energy range from $E_{0}=158 \mathrm{GeV}$ to $\sqrt{s}=13 \mathrm{TeV}$ is given in Ref. [37].

\subsection{CMS+TOTEM data}

Figure 5 shows $d n_{c h} / d \eta$ distributions based on data of the CMS+TOTEM experiment [41] for events selected using different criteria.

Figure 5a shows so-called "NSD-enhanced" $d n_{c h} / d \eta$ distribution derived for selected events with $n_{c h}>0$ in the ranges $-6.5<\eta<-5.3$ and $5.3<\eta<6.5$ in inelastic pp interactions at $\sqrt{s}=8 \mathrm{TeV}$.
Figure 5 b shows "more-forward" $d n_{c h} / d \eta$ distribution for events selected with $n_{c h}>0$ in the ranges $-6.5<\eta<-5.3$ or $5.3<\eta<6.5$.

Figure 5c shows "SD-enhanced" $d n_{c h} / d \eta$ data derived for selected events with $n_{c h}>0$ only in the ranges $-6.5<\eta<$ -5.3 or only $5.3<\eta<6.5$ at $\sqrt{s}=8 \mathrm{TeV}$. The left part of this symmetric distribution is cut off.

Figure 5d shows "more-forward" $d n_{c h} / d \eta$ data derived with displaced interaction points for selected events with $n_{c h}>0$ in the ranges $-7.0<\eta<-6.0$ or $3.7<\eta<4.8$.

Experimental statistical and systematical errors are shown by shorter and longer limits, respectively. Corresponding FANSY 2.0 QGSCPG results are also shown in Fig. 5. Experimental and simulated distributions are obviously consistent within the systematical errors.

\subsection{LHCf "neutron" and $\gamma$-ray data}

Decay-in-flight of all relatively stable particles $\left(\tau_{0}>10^{-13}\right.$ s), including $\pi, K, D$ mesons, $\Lambda, \Sigma, \Xi$ hyperons and $\Lambda_{c}^{ \pm}$ baryons, has been simulated taking into account the distance from the interaction point to the LHCf detector being as large as $140 \mathrm{~m}$ and decay probabilities for these hadrons, which are depend on particle energies and their lifetimes.

Figure 6 shows inelastic FANSY 2.0 QGSCPG and LHCf "neutron" ( $n, \bar{n}, \Lambda^{0}, \bar{\Lambda}^{0}, \mathrm{~K}_{L}^{0}$ etc.) $d \sigma / d x_{\mathrm{F}}$ cross sections at $p_{t}<0.11 x_{\mathrm{F}}(\mathrm{GeV} / \mathrm{c})$ and $\Delta \phi=360^{\circ}$ at $\sqrt{s}=7 \mathrm{TeV}$ [40].

Figure 7 shows FANSY 2.0 QGSCPG and LHCf [39] "neutron" $d \sigma / d E$ cross sections in the following pseudorapidity ranges (from top to bottom): $\eta>10.76,8.99<\eta<$ 9.22, and $8.81<\eta<8.99$, in the azimuthal-angle range $\Delta \phi=360^{\circ}$ and $\sqrt{s}=7 \mathrm{TeV}$. Results obtained with the use 


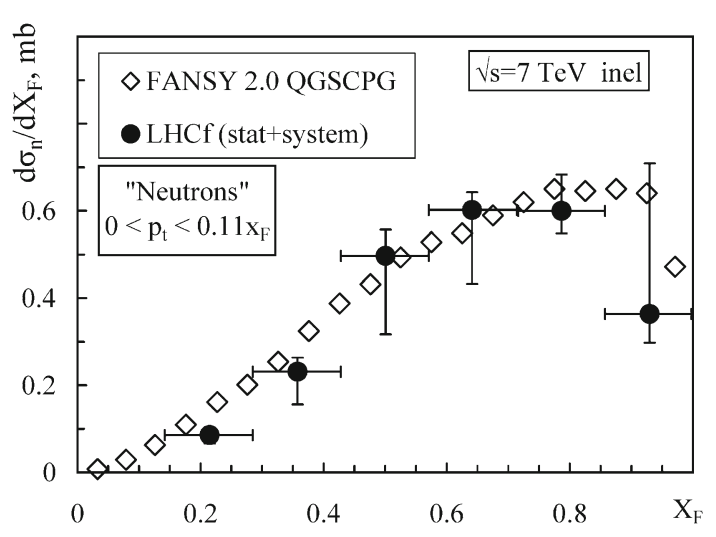

Fig. 6 LHCf [40] and FANSY 2.0 QGSCPG's $d \sigma / d x_{\mathrm{F}}$ "neutron" cross sections at $p_{t}<0.11 x_{\mathrm{F}}(\mathrm{GeV} / \mathrm{c}), \Delta \phi=360^{\circ}, \sqrt{s}=7 \mathrm{TeV}$

of QGSJETII-03 [26,27], DPMJET 3.04 [28], SIBYLL 2.1 [29-31], EPOS 1.99 [32,33], PYTHIA 8.145 [34] models are shown as well.

Figure 8 shows FANSY 2.0 and LHCf [38] $\gamma$-ray $n_{\gamma} / N_{\text {inel }}$ energy spectra at $\sqrt{s}=7 \mathrm{TeV}$ in two pseudorapidity ranges, $8.81<\eta<8.99, \Delta \phi=20^{\circ}$ (top) and $\eta>10.94, \Delta \phi=$ $360^{\circ}$ (bottom).

One can see a good agreement between the FANSY 2.0 results and collider data.

\section{Search for coplanarity at the LHC}

The LHC energy range is appropriate to study the CPG process. However, to test the above-described model in LHC experiments, it is necessary to solve a number of difficulties caused by collider's specific kinematics of experiments and a high beam luminosity. First of all, this effect can be studied only by event-by-event way, i.e., at lower collider luminosity. Second, the effect relates mainly to a large- $x_{\mathrm{F}}$ range.

The LHCf detector with characteristic sizes of $\sim 10 \mathrm{~cm}$, aimed at the study of high $x_{\mathrm{F}}$ and $\eta$ values and placed at $L=$ $140 \mathrm{~m}$ from interaction points, is capable of detecting neutral particles with relatively low $p_{t}$. However, this experiment detects, as a rule, only one particle to a few tens of interactions that makes it impossible to study azimuthal correlations of particles.

First of all, one should emphasize that the coplanarity effects are most clear and visible immediately after the CPG interaction. The following cascade development rapidly erode coplanarity effects with increasing depth of observation in the atmosphere. As a result, it can be observed only at high altitudes (in mountain or stratospheric experiments). It makes no sense to look for this phenomenon at the sea level. At mountain levels, it is necessary to separate energy flows in $\gamma$-families, not single particles only, with using a so-called decascading algorithm $[42,43]$ for the following analysis.

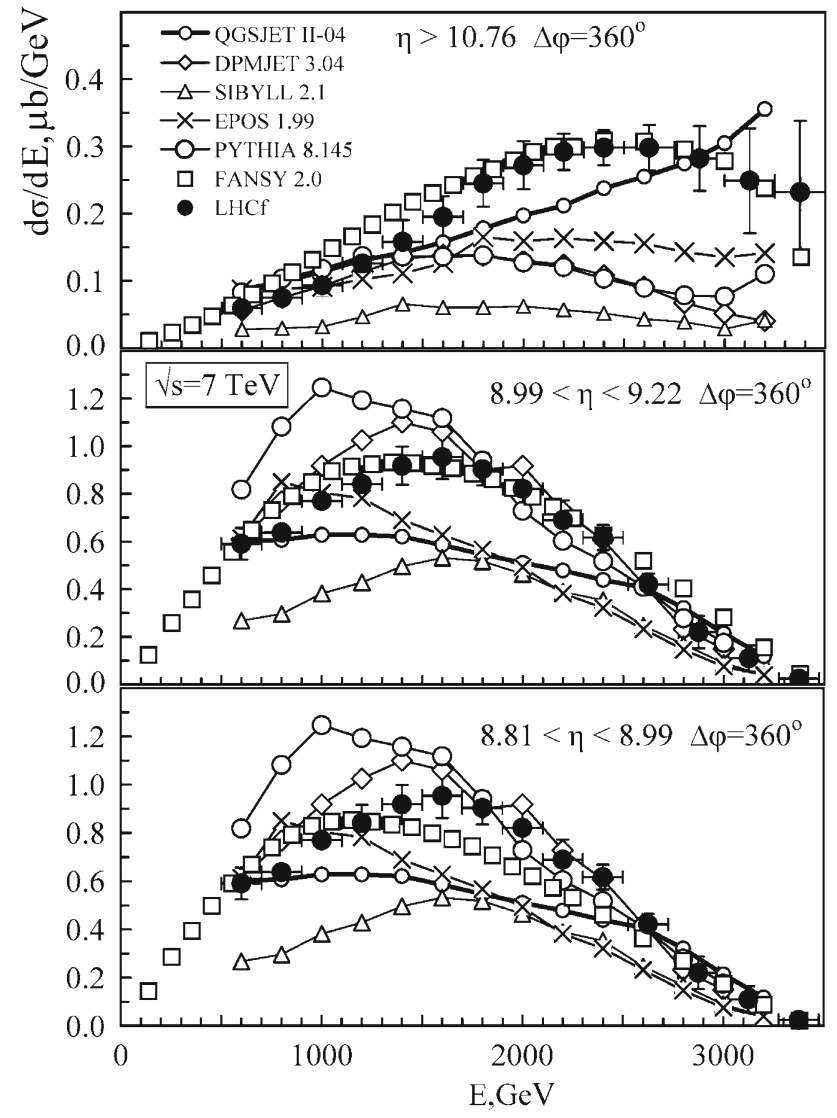

Fig. 7 Experimental LHCf (filled circles) [39] "neutron" $d \sigma / d E$ cross sections at $\eta>10.76,8.99<\eta<9.22,8.81<\eta<8.99$ (from top to bottom), $\Delta \phi=360^{\circ}, \sqrt{s}=7 \mathrm{TeV}$. Results obtained with the use of FANSY 2.0 QGSCPG ( $\square$ ), QGSJETII-03 (O) [26,27], DPMJET 3.04 $(\diamond)$ [28], SIBYLL $2.1(\triangle)$ [29-31], EPOS $1.99(\times)$ [32,33], PYTHIA $8.145(\bigcirc)[34]$ models are shown as well

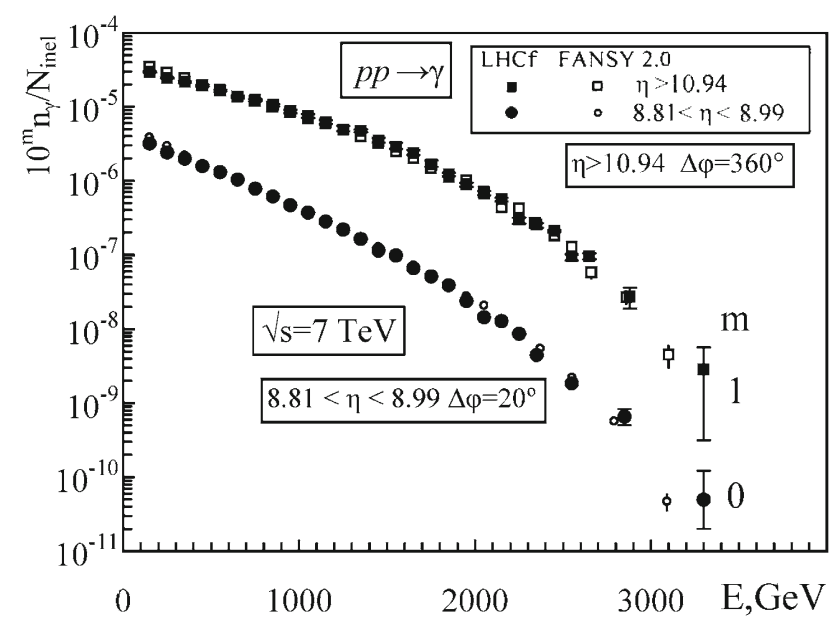

Fig. 8 LHCf [38] and FANSY $2.0 \gamma$-ray $n_{\gamma} / N_{\text {inel }} / \mathrm{GeV}$ energy spectra at $\eta>10.94, \Delta \phi=360^{\circ}$ (upper symbols) and $8.81<\eta<8.99$, $\Delta \phi=20^{\circ}$ (lower symbols) multiplied by $10^{m}$, where $m=1$ and 0 , respectively, at $\sqrt{s}=7 \mathrm{TeV}$ 
Table 2 Fractions of coplanar interactions calculated for the four most energetic stable particles, $F\left(\lambda_{4}>0.8\right)$, obtained with the FANSY 2.0 versions (QGSJ and QGSCPG) at $\sqrt{s}=7 \mathrm{TeV}$. The parameter $\lambda_{4}$ is defined by Eq. (1)

\begin{tabular}{ll}
\hline FANSY 2.0 version & $F\left(\lambda_{4}>0.8\right)$ \\
\hline QGSJ & $0.055 \pm 0.002$ \\
QGSCPG & $0.334 \pm 0.005$ \\
\hline
\end{tabular}

Second, the proton-initiated $\gamma$-ray families dominate among observable events at any, more or less, reliable PCR mass composition due to XREC's high-energy threshold of detection and, as a result, lower efficiency of nuclei in formation of families, which decreases quickly with increasing mass of primary nuclei. On the other hand, each nucleusinitiated $\gamma$-ray family is a result of superposition of separated nucleon-initiated cascades. If so, such families are characterized by a lower coplanarity in the framework of any CPG-type model.

Therefore, to analyze the coplanarity problem in the first approximation, it is advisable to consider exactly the protonproton interactions.

Table 2 shows fractions, $F\left(\lambda_{4}>0.8\right)$, of interactions with four aligned tracks of stable MEPs on an imaginary target plane calculated with FANSY 2.0's traditional (QGSJ) and coplanar generation (QGSCPG) versions at $\sqrt{s}=7 \mathrm{TeV}$. Definition of $\lambda_{4}$ is determined by Eq. (1). One can see a significant difference in results.

The CASTOR experiment [44] seems to be promising. Its operation pseudorapidity range is $5.25<\eta<6.5$, while some CPG effects could manifest themselves in this range (at least, in the framework of FANSY 2.0).

Let us simplistically estimate potentialities of the CASTOR taking into account that much more detailed simulation is undoubtedly required for serious and quantitative analysis of this problem.

Figure 9 shows a simplified cross section scheme of CASTOR and an example of detection of one coplanar interaction. The detector consists of 16 segments and is divided in the middle by a vertical gap. Particles are assumed to be detected if their pseudorapidity values are in the range of $5.3<\eta<6.5$ and they do not fall into the vertical gap.

Black circles in Fig. 9 show tracks of particles. The larger the circle size, the higher the energy of the particle. Highenergy particles show a tendency to some coplanarity. Lowenergy particles form a more or less azimuthally symmetric halo.

To analyze observed interactions, energy values "measured" in each of the segments, $E_{i}$, are used. Events with total energy $\sum E_{i} \geq 1 \mathrm{TeV}$ are only analyzed, in which the number of segments, $N_{s}$, with measured energy $E_{i}>$ $E_{i \min }=100 \mathrm{GeV}$ is equal to or greater than the minimum

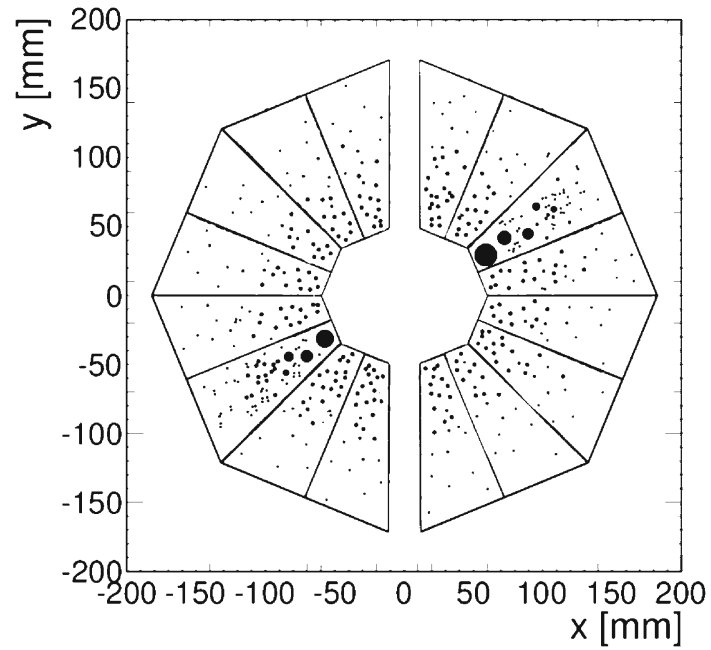

Fig. 9 CASTOR's simplified cross section scheme with an example of detection of one coplanar interaction. The larger the circle size, the higher the energy of the particle

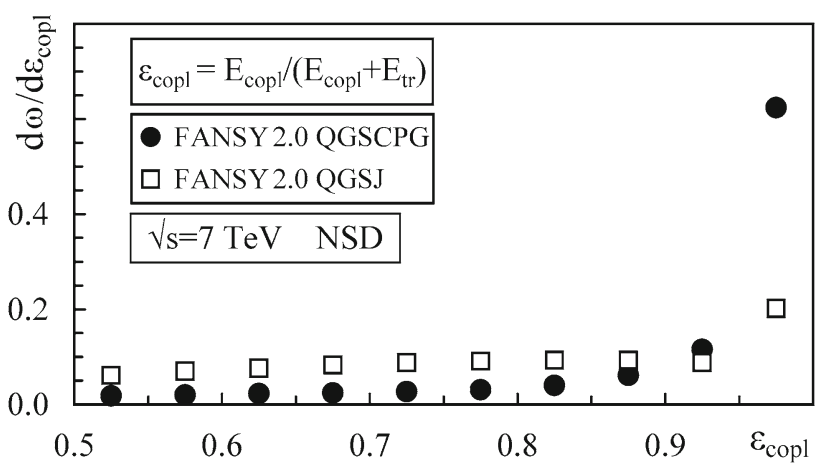

Fig. 10 Probability $d \omega / d \varepsilon_{\text {copl }}$ distributions predicted by FANSY 2.0 QGSJ and QGSCPG versions for the CASTOR detector

value, $N_{s} \geqslant N_{s \min }=2$. The first number is assigned to a segment with the maximum release of energy, $E_{\max }$. Here and below, $E_{1}=E_{\max }, E_{\mathrm{copl}}=E_{1}+E_{9} ; E_{t r}=E_{5}+E_{13}$, i.e., $E_{t r}$ is the energy measured in 9 th and 13th segments, perpendicular to the first segment. A simple parameter is applied, namely, $\varepsilon_{\text {copl }}=E_{\text {copl }} /\left(E_{\text {copl }}+E_{t r}\right)$, which characterizes the event coplanarity degree. The degree of event coplanarity is maximum at $\varepsilon_{\text {copl }}=1$.

Figure 10 shows $d \omega / d \varepsilon_{\text {copl }}$ probability distributions (normalized to unity) reproduced with FANSY 2.0 QGSJ and QGSCPG versions. Obviously, different FANSY 2.0 versions predict different probability magnitudes to observe coplanar interactions at $\varepsilon_{\text {copl }} \rightarrow 1$. Of course, it is necessary to pay our attention on fluctuations, which form some peak near $\varepsilon_{\text {copl }} \rightarrow 1$ even in the case of the traditional interactions simulated in the framework of the FANSY 2.0 QGSJ version. 


\section{Conclusion}

The FANSY 2.0 model is developed to study superhighenergy cosmic-ray "forward physics" interactions in general and the coplanar particle generation (CPG) phenomenon in particular.

At present, FANSY 2.0 includes two versions, i.e., the traditional QGSM-based QGSJ version and unconventional QGSCPG one including a CPG mode.

Comparison of results of these versions with experimental data demonstrates that the CPG process simulated with FANSY 2.0 QGSCPG version does not contradict LHC data. However, to reconcile experimental and simulated data, it is necessary to move from the primary concept of growth of transverse momenta of MEPs in the coplanarity plane to a new concept of reduction of transverse momentum component directed normally to the coplanarity plane so that the average $p_{t}$ values remain traditional. This new concept could be most naturally related to the speculative "crystal word" hypothesis only.

Some increase of the average transverse momentum of MEPs, $\left\langle p_{t}^{\text {copl }}\left(y>y_{t h r}^{c o p l}\right)\right\rangle$, is not excluded.

A model assuming that coplanar generation process takes place in double-diffraction interactions only [18] requires a separate consideration.

It would be very desirable to test the above-described model in LHC experiments.

Data Availability Statement This manuscript has no associated data or the data will not be deposited. [Authors' comment: FANSY 2.0 data will be released after the completion of the model design, that is, the development of meson-proton and hadron-nucleus simulation algorithms and publication of relevant results.]

Open Access This article is distributed under the terms of the Creative Commons Attribution 4.0 International License (http://creativecomm ons.org/licenses/by/4.0/), which permits unrestricted use, distribution, and reproduction in any medium, provided you give appropriate credit to the original author(s) and the source, provide a link to the Creative Commons license, and indicate if changes were made. Funded by SCOAP . $^{3}$

\section{References}

1. J.R. Hörandel, W.D. Apel, J.C. Arteaga-Velazquez et al., arXiv:1711.02643v2 [astro-ph.HE] (2017)

2. A. Borisov et al. (Pamir Collaboration), Proc. 4th Int. Symp. Very High Energy Cosmic Ray Interactions, Beijing 4-29 (1986)

3. I.P. Ivanenko et al., JETP Lett. 50, 2125 (1992)

4. V.V. Kopenkin et al., Phys. Rev. D 52, 2766 (1995)
5. The Pamir Collaboration, SINP MSU preprint 1989-67/144

6. A.S. Borisov et al., Nucl. Phys. B (Proc. Suppl.) 97, 118 (2001)

7. L. Xue et al., Proc. 26th Int. Cosmic Ray Conf., Salt Lake City 1 127 (1999)

8. A.V. Apanasenko et al., Proc. 15th Int. Cosmic Ray Conf., Plovdiv (1977) 7220

9. A.K. Managadze et al., Proc. 27th Int. Cosmic Ray Conf., Hamburg $11426(2001)$

10. J.N. Capdevielle, J. Phys. G 14, 503 (1988)

11. J.N. Capdevielle et al., Proc. 30th Int. Cosmic Ray Conf., Merida, HE, no. 120 (2007)

12. R.A. Mukhamedshin, JHEP 05, 049 (2005)

13. R.A. Mukhamedshin, Nucl. Phys. B (Proc. Suppl.) 196C, 98 (2009)

14. R.A. Mukhamedshin, Eur. Phys. J. C 60, 345 (2009)

15. A.K. Managadze et al., Proc. 29th Int. Cosmic Ray Conf., Pune 9 $81(2005)$

16. A.K. Managadze et al., Phys. Atomic Nuclei 70(1), 184 (2007)

17. T. Wibig. arXiv:hep-ph/0003230

18. I.I. Royzen, Mod. Phys. Lett. A 9(38), 3517 (1994)

19. J.N. Capdevielle, Nucl. Phys. B (Proc. Suppl.) 175-176, 137 (2008)

20. T.S. Yuldashbaev et al., Nuovo Cim. 24C, 569 (2001)

21. R.A. Mukhamedshin, Nucl. Phys. B (Proc. Suppl.) 75A, 141 (1999)

22. L.A. Anchordoqui et al.. arXiv: 1003.5914 [hep-ph]

23. I.M. Dremin, JETP Lett. 30, 140-147 (1979)

24. E. Fermi, Prog. Theor. Phys. 5, 570 (1950)

25. R. Hagedorn, Il Nouvo Cim. 15, 434 (1960)

26. S. Ostapchenko, Phys. Rev. D 74, 014026 (2006)

27. S. Ostapchenko, Phys. Lett. B 636, 40 (2006)

28. F.W. Bopp et al., Phys. Rev. C 77, 014904 (2008)

29. J. Engel et al., Phys. Rev. D 46, 5013 (1992)

30. R. Engel et al., Proc. 26th Int. Cosmic Ray Conf., Salt Lake City 1 415 (1999)

31. E.J. Ahn et al., Phys. Rev. D 80, 094003 (2009). 0906.4113

32. K. Werner et al., Phys. Rev. C 74, 044902 (2006). arXiv:hep-ph/0506232

33. T. Pierog, K. Werner, Nucl. Phys. Proc. Suppl. 196, 102 (2009)

34. T. Sjostrand et al., Introduction to PYTHIA 8.1. Comput. Phys. Commun. 178, 852 (2008)

35. The CMS Collaboration, arXiv:1009.4122v1 [hep-ex]

36. J. Kempa, R. Mukhamedshin, M. Tamada, J. Phys. Conf. Ser. 409, 012109 (2013)

37. R.A. Mukhamedshin. FANSY 2.0: a Monte Carlo tool for study of superhigh-energy cosmic-ray interactions (is sent to Eur. Phys. J. Plus)

38. O. Adriani et al., Phys. Lett. B 703, 128 (2011)

39. O. Adriani et al. arXiv:1503.03505v1 [hep-ex]

40. K. Kawade, $\mathrm{PhD}$ thesis, CERN-THESIS-2014-315

41. The CMS Collaboration and TOTEM Collaboration, Eur.Phys.J. C 74 (2014) 2053; arXiv:1405.0722v1

42. A.S. Borisov et al., Nucl. Phys. B (Proc. Suppl.) 75A, 144 (1999)

43. Pamir Collaboration, Trudy FIAN 154, 3 (1985). (in Russian)

44. P. Gunnellini (on behalf of the CMS collaboration). arXiv:1304.2943 [physics.ins-det] 